\title{
An Interdisciplinary Approach to Metastatic Pancreatic Cancer and Comorbid Opioid Use Disorder Treatment Within a VA Health Care System
}

\author{
Andrea Ruskin, MD; Caroline G. Falker, MD; Ellen L. Edens, MD, MPE; and Margaret R. Bauer, PhD
}

A multidisciplinary approach provided safe and feasible cancer treatment in a patient with advanced pancreatic cancer and coexisting active substance use disorder.

\begin{abstract}
Andrea Ruskin is Medical Director Palliative Care; Caroline Falker is

Physician Internal Medicine; and Margaret

Bauer is Psychologist, Palliative Care Team and Health Psychology Service; all at Veterans Affairs Connecticut Healthcare System in West Haven. Ellen Edens is Associate Professor of Psychiatry, Yale University School of Medicine in New Haven, Connecticut.

Correspondence: Andrea Ruskin (aruskin@optonline.net)
\end{abstract}

Fed Pract. 2021;8(suppl 3) Published online August 15. doi:10.12788/fp.0160

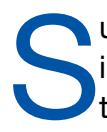
ubstance use disorders (SUDs) are an important but understudied aspect of treating patients diagnosed with cancer. Substance use can affect cancer treatment outcomes, including morbidity and mortality. ${ }^{1,2}$ Additionally, patients with cancer and SUD may have unique psychosocial needs that require close attention and management. There is a paucity of data regarding the best approach to treating such patients. For example, cocaine use may increase the cardiovascular and hematologic risk of some traditional chemotherapy agents. ${ }^{3,4}$ Newer targeted agents and immunotherapies remain understudied with respect to SUD risk.

Although the US Department of Veterans Affairs (VA) has established helpful clinical practice guidelines for the treatment of SUD, there are no guidelines for treating patients with SUD and cancer. ${ }^{5}$ Clinicians have limited confidence in treatment approach, and treatment is inconsistent among oncologists nationwide even within the same practice. Furthermore, it can be challenging to safely prescribe opioids for cancerrelated pain in individuals with SUD. There is a high risk of SUD and mental health disorders in veterans, making this population particularly vulnerable. We report a case of a male with metastatic pancreatic cancer, severe opioid use disorder (OUD) and moderate cocaine use disorder (CUD) who received pain management and cancer treatment under the direction of a multidisciplinary team approach.

\section{CASE REPORT}

A 63-year-old male with a medical history of HIV treated with highly active antiretroviral therapy (HAART), compensated cirrhosis, severe OUD, moderate CUD, and sedative use disorder in sustained remission was admitted to the West
Haven campus of the VA Connecticut Healthcare System (VACHS) with abdominal pain, weight loss and fatigue. He used heroin 1 month prior to his admission and reported regular cocaine and marijuana use (Table 1). He was diagnosed with HIV in 1989, and his medical history included herpes zoster and oral candidiasis but no other opportunistic infections. Several months prior to this admission, he had an undetectable viral load and CD4 count of 688 .

At the time of this admission, the patient was adherent to methadone treatment. He reported increased abdominal pain. Computed tomography (CT) showed a $2.4-\mathrm{cm}$ mass in the pancreatic uncinate process, multiple liver metastases, retroperitoneal lymphadenopathy, and small lung nodules. A CT-guided liver biopsy showed adenocarcinoma consistent with a primary cancer of the pancreas. Given the complexity of the case, a multidisciplinary team approach was used to treat his cancer and the sequelae safely, including the oncology team, community living center team, palliative care team, and interprofessional opioid reassessment clinic team (ORC).

\section{Cancer Treatment}

Chemotherapy with FOLFIRINOX (leucovorin calcium, fluorouracil, irinotecan hydrochloride, and oxaliplatin) was recommended. The first cycle of treatment originally was planned for the outpatient setting, and a peripherally inserted central catheter (PICC) line was placed. However, after a urine toxicology test was positive for cocaine, the PICC line was removed due to concern for possible use of PICC line for nonprescribed substance use. The patient expressed suicidal ideation at the time and was admitted for psychiatric consult and pain control. Cycle 1 FOLFIRINOX was started during this admission. A PICC line was again put in place and then 
TABLE 1 Diagnostic Criteria for Substance Use Disorder and Case Diagnoses ${ }^{14}$

\begin{tabular}{|c|c|c|}
\hline DSM-5 Substance Use Disorder Diagnostic Criteriaa & Modifiers & Current Case Diagnoses \\
\hline $\begin{array}{l}\text { Within a 12-month period: } \\
\text { Taken in larger amount/longer time } \\
\text { Unsuccessful attempts to cut down/control use } \\
\text { Significant time spent obtaining/recovering from substance } \\
\text { Cravings } \\
\text { Failure fulfilling home/work/school obligations } \\
\text { Social/interpersonal problems related to use } \\
\text { Social/occupational/recreational activities given up due to use } \\
\text { Physically hazardous use } \\
\text { Continues use despite knowledge of problems related to use } \\
\text { Drug tolerance (increased amount/diminished effect of same amount) } \\
\text { Withdrawal }\end{array}$ & $\begin{array}{l}\text { Severity } \\
\text { Mild ( } 2-3 \text { symptoms); } \\
\text { Moderate ( } 4-5 \text { symptoms); } \\
\text { Severe ( } 5 \text { symptoms); } \\
\text { Remission } \\
\text { Early (no criteria for }>3 \text { mo } \\
\text { but }<12 \text { mo); } \\
\text { Sustained (no criteria for } \\
>12 \text { mo) }\end{array}$ & $\begin{array}{l}\text { Opioid use disorder, } \\
\text { Severe cocaine use disorder, } \\
\text { Moderate sedative use disorder, } \\
\text { In sustained remission }\end{array}$ \\
\hline
\end{tabular}

Abbreviation: DSM-5, Diagnostic and Statistical Manual of Mental Disorders, 5th edition.

${ }^{a}$ Critieria can be applied for alcohol, cannabis, hallucinogens, inhalants, opioids, sedatives/hypnotics/anxiolytics, stimulants, and tobacco.

bWithdrawal not a criteria for cannabis, inhalants, and hallucinogens.

removed before discharge. A celiac plexus block was performed several days after this admission for pain control.

Given concern about cocaine use increasing the risk of cardiac toxicity with FOLFIRINOX treatment, treating providers sconsulted with the community living center (CLC) about possible admission for future chemotherapy administration and pain management. The CLC at VACHS has 38 beds for rehabilitation, long-term care, and hospice with the mission to restore each veteran to his or her highest level of wellbeing. After discussion with this patient and CLC staff, he agreed to a CLC admission. The patient agreed to remain in the facility, wear a secure care device, and not leave without staff accompaniment. He was able to obtain a 2-hour pass to pay bills and rent. During the 2 months he was admitted to the CLC he would present to the VACHS Cancer Center for chemotherapy every 2 weeks. He completed 6 cycles of chemotherapy while admitted. During the admission, he was transferred to active medical service for 2 days for fever and malaise, and then returned to the CLC. The patient elected to leave the CLC after 2 months as the inability to see close friends was interfering with his quality of life.

Upon being discharged from the CLC, shared decision making took place with the patient to establish a new treatment plan. In collaboration with the patient, a plan was made to admit him every 2 weeks for continued chemotherapy. A PICC line was placed on each day of admission and removed prior to discharge. It was also agreed that treatment would be delayed if a urine drug test was positive for cocaine on the morning of admission. The patient was also seen by ORC every 2 weeks after being discharged from the CLC.

Imaging after cycle 6 showed decreased size of liver metastases, retroperitoneal lymph nodes, and pancreas mass. Cancer antigen 19-9 (CA19-9) tumor marker was reduced from $3513 \mathrm{U} / \mathrm{mL}$ pretreatment to $50 \mathrm{U} / \mathrm{mL}$ after cycle 7. Chemotherapy cycle 7 was delayed 6 days due to active cocaine and heroin use. A repeat urine was obtained several days later, which was negative for cocaine, and he was admitted for cycle 7 chemotherapy. Using this treatment approach of admissions for every cycle, the patient was able to receive 11 cycles of FOLFIRINOX with clinical benefit.

\section{Palliative Care/Pain Management}

Safely treating the patient's malignant pain in the context of his OUD was critically important. In order to do this the palliative care team worked closely alongside ORC, is a multidisciplinary team consisting of health care providers (HCPs) from addiction psychiatry, internal medicine, health psychology and pharmacy who are consulted to evaluate veterans' current opioid regimens and make recommendations to optimize both safety and efficacy. ORC followed this particular veteran as an outpatient and consulted on pain issues during his admission. They recommended the continuation of methadone at $120 \mathrm{mg}$ daily and increased oral oxycodone to $30 \mathrm{mg}$ every 6 hours, and then further increased to 
$45 \mathrm{mg}$ every 6 hours. He continued to have increased pain despite higher doses of oxycodone, and pain medication was changed to oral hydromorphone $28 \mathrm{mg}$ every 6 hours with the continuation of methadone. ORC and the palliative care team obtained consent from the veteran and a release of Information form signed by the patient to contact his community methadone clinic for further collaboration around pain management throughout the time caring for the veteran.

Even with improvement in disease based on imaging and tumor markers, opioid medications could not be decreased in this case. This is likely in part due to the multidimensional nature of pain. Careful assessment of the biologic, emotional, social, and spiritual contributors to pain is needed in the management of pain, especially at end of life. ${ }^{6}$ Nonpharmacologic pain management strategies used in this case included a transcutaneous electrical nerve stimulation unit, moist heat, celiac plexus block, and emotional support.

\section{Psychosocial Issues/Substance Use}

Psychosocial support for the patient was provided by the interdisciplinary palliative care team and the ORC team in both the inpatient and outpatient settings. Despite efforts from case management to get the veteran home services once discharged from the CLC, he declined repeatedly. Thus, the CLC social worker obtained a guardian alert for the veteran on discharge.

Close outpatient follow-up for medical and psychosocial support was very critical. When an outpatient, the veteran was scheduled for biweekly appointments with palliative care or ORC. When admitted to the hospital, the palliative care team medical director and psychologist conducted joint visits with him. Although he denied depressed mood and anxiety throughout his treatment, he often reflected on regrets that he had as he faced the end of his life. Specifically, he shared thoughts about being estranged from his surviving brother given his long struggle with substance use. Although he did not think a relationship was possible with his brother at the end of life, he still cared deeply for him and wanted to make him aware of his pancreatic cancer diagnosis. This was particularly important to him because their late brother had also died of pancreatic cancer. It was the patient's wish at the end of his life to alert his surviving brother of his diagnosis so he and his children could get adequate screening throughout their lives. Although he had spoken of this desire often, it wasn't until his disease progressed and he elected to transition to hospice that he felt ready to write the letter. The palliative care team assisted the veteran in writing and mailing a letter to his brother informing him of his diagnosis and transition to hospice as well as communicating that his brother and his family had been in his thoughts at the end of his life. The patient's brother received this letter and with assistance from the CLC social worker made arrangements to visit the veteran at bedside at the inpatient CLC hospice unit the final days of his life.

\section{DISCUSSION}

There are very little data on the safety of cancer-directed therapy in patients with active SUD. The limited studies that have been done showed conflicting results.

A retrospective study among women with co-occurring SUD and locally advanced cervical cancer who were undergoing primary radiation therapy found that SUD was not associated with a difference in toxicity or survival outcomes. ${ }^{7}$ However, other research suggests that SUD may be associated with an increase in all-cause mortality as well as other adverse outcomes for patients and health care systems (eg, emergency department visits, hospitalizations). ${ }^{8}$ A retrospective study of patients with a history of SUD and nonsmall cell lung cancer showed that these patients had higher rates of depression, less family support, increased rates of missed appointments, more emergency department visits and more hospitalizations. ${ }^{9}$ Patients with chronic myeloid leukemia or myelodysplastic syndromes who had longterm cocaine use had a 6-fold increased risk of death, which was not found in patients who had long-term alcohol or marijuana use. ${ }^{2}$

The limited data highlight the need for careful consideration of ways to mitigate potentially adverse outcomes in this population while still providing clinically indicated cancer treatment. Integrated VA health care systems provide unique resources that can maximize veteran safety during cancer treatment. Utilization of VA resources and close interdisciplinary collaboration across VA HCPs can help to ensure equitable access to state-of-the-art cancer therapies for veterans with comorbid SUD. 
TABLE 2 Considerations for Working With Individuals With Active Substance Use and Complex Medical Conditions

\begin{tabular}{ll} 
Levels & Considerations \\
\hline $\begin{array}{l}\text { Health care } \\
\text { provider }\end{array}$ & $\begin{array}{l}\text { Obtain appropriate SUD history } \\
\text { Review literature to determine risks for interactions between recommended medical treatment and } \\
\text { substance of use } \\
\text { Engage in motivational interviewing to resolve ambivalence toward change; consider medications } \\
\text { for SUD } \\
\text { Informed consent around treatment, including clear education to patient about risks/benefits of } \\
\text { treatment with current use } \\
\text { Shared decision making around treatment } \\
\text { Employ risk reduction strategies (eg, setting of treatment, abstinence before treatment days, } \\
\text { naloxone prescription, short opioid refills) } \\
\text { Interprofessional collaboration with other providers to support patient throughout treatment } \\
\text { Utilization of interprofessional provider meetings to create unified efforts }\end{array}$ \\
& $\begin{array}{l}\text { Motivational interviewing and shared decision making education for specialty health care providers } \\
\text { SUD diagnoses and treatment education for specialty health care providers }\end{array}$ \\
Health & VA mental health/SUD resources education for specialty health care providers \\
systems & $\begin{array}{l}\text { Embedded psychosocial support staff in VA Cancer Centers, including psychology, palliative care, } \\
\text { and social work. }\end{array}$ \\
Abbreviations: SUD & substance use disorder; VA, uS Department of Veterans Affairs.
\end{tabular}

Abbreviations: SUD, substance use disorder; VA, US Department of Veterans Affairs.

\section{VA Services for Patients With Comorbidities}

This case highlights several distinct aspects of VA health care that make it possible to safely treat individuals with complex comorbidities. One important aspect of this was collaboration with the CLC to admit the veteran for his initial treatment after a positive cocaine test. CLC admission was nonpunitive and allowed ongoing involvement in the VA community. This provided an essential, safe, and structured environment in which 6 cycles of chemotherapy could be delivered.

Although the patient left the CLC after 2 months due to floor restrictions negatively impacting his quality of life and ability to spend time with close friends, several important events occurred during this stay. First, the patient established close relationships with the CLC staff and the palliative care team; both groups followed him throughout his inpatient and outpatient care. These relationships proved essential throughout his care as they were the foundation of difficult conversations about substance use, treatment adherence, and eventually, transition to hospice.

In addition, the opportunity to administer 6 cycles of chemotherapy at the CLC was enough to lead to clinical benefit and radiographic response to treatment. Clinical benefits while in the CLC included maintenance of a good appetite, 15-lb weight gain and preserved performance status (ECOG [Eastern Cooperative Group]-1), which allowed him to actively participate in multiple social and recreational activities while in the CLC. From early conversations, this patient was clear that he wanted treatment as long as his life could be prolonged with good quality of life. Having evidence of the benefit of treatment, at least initially, increased the patient's confidence in treatment. There were a few conversations when the challenges of treatment mounted (eg, pain, needs for abstinence from cocaine prior to admission for chemotherapy, frequent doctor appointments), and the patient would remind himself of these data to recommit himself to treatment. The opportunity to admit him to the inpatient VA facility, including bed availability for 3 days during his treatment once he left the CLC was important. This plan to admit the patient following a negative urine toxicology test for cocaine was made collaboratively with the veteran and the oncology and palliative care teams. The plan allowed the patient to achieve his treatment goals while maintaining his safety and reducing theoretical cardiac toxicities with his cancer treatment.

Finally, the availability of a multidisciplinary team approach including palliative care, oncology, psychology, addiction medicine and addiction psychiatry, was critical for addressing the veteran's malignant pain. Palliative care 
worked in close collaboration with the ORC to prescribe and renew pain medications. ORC offered ongoing consultation on pain management in the context of OUD. As the veteran's cancer progressed and functional decline prohibited his daily attendance at the community methadone clinic, palliative care and ORC met with the methadone clinic to arrange a less frequent methadone pickup schedule (the patient previously needed daily pickup). Non-VA settings may not have access to these resources to safely treat the biopsychosocial issues that arise in complex cases.

\section{Substance Use and Cancer Treatments}

This case raises several critical questions for oncologic care. Cocaine and fluorouracil are both associated with cardiotoxicity, and many oncologists would not feel it is safe to administer a regimen containing fluorouracil to a patient with active cocaine use. The $\mathrm{Na}$ tional Comprehensive Cancer Network (NCCN) panel recommends FOLFIRINOX as a preferred category 1 recommendation for first-line treatment of patients with advanced pancreas cancer with good performance status. ${ }^{10}$ This recommendation is based on the PRODIGE trial, which has shown improved overall survival (OS): 11.1 vs 6.8 months for patients who received single-agent gemcitabine..$^{11}$ If patients are not candidates for FOLFIRINOX and have good performance status, the NCCN recommends gemcitabine plus albumin-bound paclitaxel with category 1 level of evidence based on the IMPACT trial, which showed improvement in OS (8.7 vs 6.6 months compared with single-agent gemcitabine). ${ }^{12}$

Some oncologists may have additional concerns administering fluorouracil treatment alternatives (such as gemcitabine and albumin-bound paclitaxel) to individuals with active SUD because of concerns about altered mental status impacting the ability to report important adverse effects. In the absence of sufficient data, HCPs must determine whether they feel it is safe to administer these agents in individuals with active cocaine use. However, denying these patients the possible benefits of standard-of-care life-prolonging therapies without established data raises concerns regarding the ethics of such practices. There is concern that the stigma surrounding cocaine use might contribute to withholding treatment, while treatment is continued for individuals taking pre- scribed stimulant medications that also have cardiotoxicity risks. VA health care facilities are uniquely situated to use all available resources to address these issues using interprofessional patient-centered care and determine the most optimal treatment based on a risk/benefit discussion between the patient and the HCP.

Similarly, this case also raised questions among HCPs about the safety of using an indwelling port for treatment in a patient with SUD. In the current case there was concern about keeping in a port for a patient with a history of IV drug use; therefore, a PICC line was initiated and removed at each admission. Without guidelines in these situations, HCPs are left to weigh the risks and benefits of using a port or a PICC for individuals with recent or current substance use without formal data, which can lead to inconsistent access to care. More guidance is needed for these situations.

\section{SUD Screening}

This case begs the question of whether oncologists are adequately screening for a range of SUDs, and when they encounter an issue, how they are addressing it. Many oncologists do not receive adequate training on assessment of current or recent substance use. There are health care and systems-level practices that may increase patient safety for individuals with ongoing substance use who are undergoing cancer treatment. Training on obtaining appropriate substance use histories, motivational interviewing to resolve ambivalence about substance use in the direction of change, and shared decision making about treatment options could increase confidence in understanding and addressing substance use issues. It is also important to educate oncologists on how to address patients who return to or continued substance use during treatment. In this case the collaboration from palliative care, psychology, addiction medicine, and addiction psychiatry through the ORC was essential in assisting with ongoing assessment of substance use, guiding difficult conversations about the impact of substance use on the treatment plan, and identifying risk-mitigation strategies. Close collaboration and full utilization of all VA resources allowed this patient to receive firstline treatment for pancreatic cancer in order to reach his goal of prolonging his life while maintaining acceptable quality of life. Table 2 provides best practices for management of patients with comorbid SUD and cancer. 
More research is needed into cancer treatment for patients with SUD, especially in the current era of cancer care using novel cancer treatments leading to significantly improved survival in many cancer types. Ideally, oncologists should be routinely or consistently screening patients for substance use, including alcohol. The patient should participate in this decision-making process after being educated about the risks and benefits. These patients can be followed using a multimodal approach to increase their rates of success and improve their quality of life. Although the literature is limited and no formal guidelines are available, VA oncologists are fortunate to have a range of resources available to them to navigate these difficult cases. Veterans have elevated rates of SUD, making this a critical issue to consider in the VA. ${ }^{13}$ It is the hope that this case can highlight how to take advantage of the many VA resources in order to ensure equitable cancer care for all veterans.

\section{CONCLUSIONS}

This case demonstrates that cancer-directed treatment is safe and feasible in a patient with advanced pancreatic cancer and coexisting active SUD by using a multidisciplinary approach. The multidisciplinary team included palliative care, oncology, psychology, addiction medicine, and addiction psychiatry. Critical steps for a successful outcome include gathering history about SUD; motivational interviewing to resolve ambivalence about treatment for SUD; shared decision making about cancer treatment; and risk-reduction strategies in pain and SUD management.

Treatment advancements in many cancer types have led to significantly longer survival, and it is critical to develop safe protocols to treat patients with active SUD so they also can derive benefit from these very significant medical advancements.

\section{Acknowledgments}

Michal Rose, MD, Director of VACHS Cancer Center, and Chandrika Kumar, MD, Director of VACHS Community Living Center, for their collaboration in care for this veteran.

\section{Author disclosures}

The authors report no actual or potential conflicts of interest with regard to this article.

\section{Disclaimer}

The opinions expressed herein are those of the authors and do not necessarily reflect those of Federal
Practitioner, Frontline Medical Communications Inc., the US Government, or any of its agencies. This article may discuss unlabeled or investigational use of certain drugs. Please review the complete prescribing information for specific drugs or drug combinations - including indications, contraindications, warnings, and adverse effects-before administering pharmacologic therapy to patients.

\section{References}

1. Chang G, Meadows ME, Jones JA, Antin JH, Orav EJ. Substance use and survival after treatment for chronic myelogenous leukemia (CML) or myelodysplastic syndrome (MDS). Am J Drug Alcohol Ab. 2010;36(1):1-6. doi:10.3109/00952990903490758

2. Stagno S, Busby K, Shapiro A, Kotz M. Patients at risk: addressing addiction in patients undergoing hematopoietic SCT. Bone Marrow Transplant. 2008;42(4):221-226. doi:10.1038/bmt.2008.211

3. Arora NP. Cutaneous vasculopathy and neutropenia associated with levamisole-adulterated cocaine. Am J Med Sci. 2013;345(1):45-51. doi:10.1097/MAJ.0b013e31825b2b50

4. Schwartz BG, Rezkalla S, Kloner RA. Cardiovascular effects of cocaine. Circulation. 2010;122(24):2558-2569. doi:10.1161/CIRCULATIONAHA.110.940569

5. US Department of Veterans Affairs, US Department of Defense. VA/DoD clinical practice guideline for the management of substance use disorders. Published 2015. Accessed July 8, 2021. https://www.healthquality.va.gov /guidelines/MH/sud/VADODSUDCPGRevised22216.pdf

6. Mehta A, Chan LS. Understanding of the concept of "total pain": a prerequisite for pain control. J Hosp Palliat Nurs. 2008;10(1):26-32. doi:10.1097/01.NJH.0000306714.50539.1a

7. Rubinsak LA, Terplan M, Martin CE, Fields EC, McGuire WP, Temkin SM. Co-occurring substance use disorder: The impact on treatment adherence in women with locally advanced cervical cancer. Gynecol Oncol Rep. 2019;28:116-119. Published 2019 Mar 27. doi:10.1016/j.gore.2019.03.016

8. Chhatre S, Metzger DS, Malkowicz SB, Woody G, Jayadevappa R. Substance use disorder and its effects on outcomes in men with advanced-stage prostate cancer. Cancer. 2014;120(21):3338-3345. doi:10.1002/cncr.28861

9. Concannon K, Thayer JH, Hicks R, et al. Outcomes among patients with a history of substance abuse in non-small cell lung cancer: a county hospital experience. J Clin Onc. 2019;37(15)(suppl):e20031-e20031. doi:10.1200/JCO.2019.37.15

10. National Comprehensive Cancer Network. NCCN clinical practice guidelines in oncology: pancreatic adenocarcinoma. Version 2.2021. Updated February 25, 2021. Accessed July 8, 2021. https://www.nccn.org/professionals /physician_gls/pdf/pancreatic.pdf

11. Conroy T, Desseigne F, Ychou M, et al. FOLFIRINOX versus gemcitabine for metastatic pancreatic cancer. N Engl J Med. 2011;364(19):1817-1825. doi:10.1056/NEJMoa1011923

12. Von Hoff DD, Ervin T, Arena FP, et al. Increased survival in pancreatic cancer with nab-paclitaxel plus gemcitabine. N Engl J Med. 2013;369(18):1691-1703. doi:10.1056/NEJMoa1304369

13. Seal KH, Cohen G, Waldrop A, Cohen BE, Maguen $S$, Ren L. Substance use disorders in Iraq and Afghanistan veterans in VA healthcare, 2001-2010: Implications for screening, diagnosis and treatment. Drug Alcohol Depend. 2011;116(1-3):93-101. doi:10.1016/j.drugalcdep.2010.11.027

14. American Psychiatric Association. Diagnostic and Statistical Manual of Mental Disorders, 5th ed. American Psychiatric Association; 2013. 